\title{
Effect of Autonomic Cardiac Modulation on Speech Perception in Noise
}

\author{
Kang Pei ${ }^{1}$, Fei Chen ${ }^{1}$, Dingchang Zheng ${ }^{2}$ \\ ${ }^{1}$ Department of Electrical and Electronic Engineering, Southern University of Science and \\ Technology, Shenzhen, China \\ ${ }^{2}$ Health and Wellbeing Academy, Anglia Ruskin University, Chelmsford, UK
}

\begin{abstract}
Recognizing speech in noise is affected by many physiological, psychological and environmental modulation mechanisms. The present study aimed to examine the effect of autonomic cardiac modulation on speech perception in noise (SPIN) by investigating how cardiac activity with heart rate (HR) change influences SPIN.

Ten young and normal-hearing subjects were studied. There were 4 sessions for each subject. They were firstly given a 5-min rest for recording baseline $H R$. They were then asked to recognize a list of 20 sentences corrupted by 2-talker babble masker at -10 $d B$ signal-to-noise ratio. The SPIN score was measured, as well as the HR which reflects the influence of noise on cardiac activity. Using the same speech recognition protocol, in section 3 and 4, SPIN and HR were measured immediately and 10 mins after each subject was asked to cycle for 5-7 minutes to experience a moderate amount of exercise.

Experimental results showed that, after moderate exercise of bicycle-riding, most participants had better SPIN score in comparison with that measured before exercise (49.7\% vs $42.5 \%)$. After the recovery to a normal resting cardiac activity, the SPIN performance of $49.9 \%$ was significantly better than the normal level at resting cardiac activity $(p<0.05)$.

In summary, the present study quantitatively demonstrated the correlation between improved SPIN performance and autonomic cardiac activity.
\end{abstract}

\section{Introduction}

Noise is a type of unwanted sound, which makes listeners hard to concentrate on speech perception or communicate in their daily life. Noisy environment may cause hearing loss. The disturbance of noise in speech perception is mainly attributed to two types of masking: energetic masking and information masking [1]. Babble noise, which is very common in our life, contains both types of masking.

Recognizing speech in noise requires great efforts for cognitive concentration. So the importance of physiological and psychological state of human body, which directly or indirectly determines the excitement level of brain, is worthy investigation. On the other hand, autonomic cardiac modulation manipulates human body both physiologically and psychologically, e.g., circulation in brain. Earlier work has shown that autonomic cardiac modulation has a strong relationship with human emotion state [2], the motor function of human body [3], sleep [4], etc.

Noise, whether it is either detectable or undetectable, also affects human cardiovascular health [5]. Although it is widely accepted that noise interference affects both speech perception in noise (SPIN) and cardiovascular activity, there is still little information about the interaction between autonomic cardiac activity and SPIN. Specifically, although many studies have examined factors that affect SPIN, such as age [6], the activity of cells in auditory cortex [7] and visual information [8], little has been done to examine the effect of autonomic cardiac modulation on SPIN.

This study aimed to examine the effect of autonomic cardiac modulation on SPIN. More specifically, this work aimed to investigate how cardiac activity with increased HR influences SPIN.

\section{Method}

\subsection{Subjects}

Ten young healthy subjects ( 2 females and 8 males; aged from 19 to 23 years) with normal-hearing participated in the experiment. This study was carried out according to the Declaration of Helsinki of the world Medical Association, and was approved by university ethics committee. Informed and written consent was obtained from all subjects. Anonymised data was analysed. 


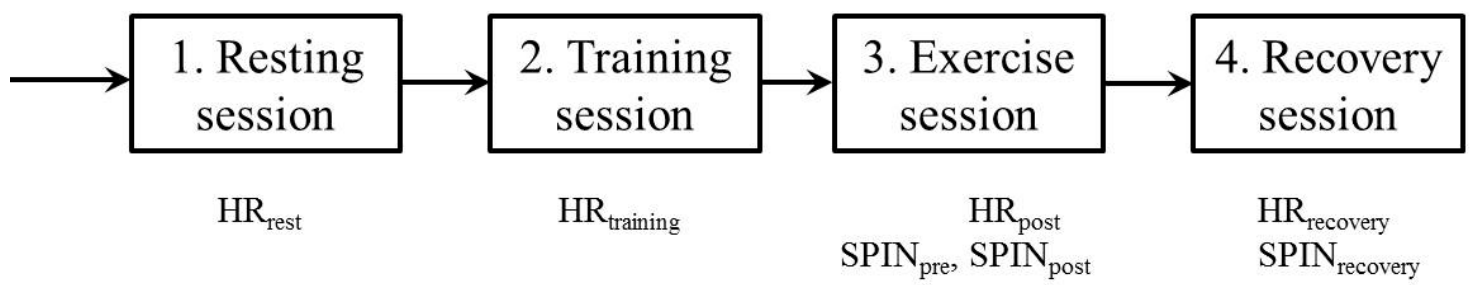

Figure 1. Block diagram of experiment procedure.

\subsection{Experiment set up}

The experiment was conducted in an acoustically and electrically shielded chamber. For each subject, two physiological parameters (HR and SPIN score) were obtained in the experiment.

The Finometer device was used to measure each participant's HR, and an exercise bicycle was utilized to do exercise in order to change his/her HR. Note that the exercise load of riding bicycle was set to a moderate level and to 5-7 min.

To measure SPIN score, test stimuli were played to each participant binaurally through a circumaural headphone at a comfortable listening level. Participants were required to repeat as many words as they could recognize. For the listening experiment, a simple custom-designed software interface was designed for each participant to control the auditory delivery of the processed stimuli. An online tester was used to score his/her response.

The speech material consisted of sentences extracted from the Mandarin Hearing in Noise Test (MHINT) database [9]. MHINT corpus has a total of 12 lists, and each list has 20 sentences (10 keywords in each sentence). A two-talker babble (2-talker, with two male voices) were used to corrupt test sentences at a signalto-noise ratio (SNR) of $-10 \mathrm{~dB}$, which was chosen by a pilot study to avoid the ceiling/floor effect in speech recognition.

\subsection{Experiment protocol}

As shown in Figure 1, the experiment consisted of four sessions:

1) Resting session: In this session, each participant was asked to have a rest for 5 mins. During this period, his/her HR was recorded and averaged, which was used as baseline HR for each participant, and noted as $\mathrm{HR}_{\text {rest. }}$.

2) Training session: Each participant attended a 10-min training session to be familiar with noise-masked speech and the procedure of listening experiment. During the training session, the subject listened to forty noise-masked speech, and his/her HR was measured and averaged, and noted as $\mathrm{HR}_{\text {training. }}$

3) Exercise session: Two speech recognition tests were performed, with a 5-7 min exercise on bicycle in between. The SPIN scores of the two speech recognition tests were noted as SPIN pre and SPIN post, indicating the scores before and after exercise, respectively. The average HR after exercise, noted as $\mathrm{HR}_{\text {post, }}$ was also recorded when collecting SPIN $_{\text {post. }}$

4) Recovery session: Each participant had a 10-min rest after the exercise. A speech recognition test was then conducted, and the HR was recorded simultaneously. The SPIN score and average HR were noted as SPIN $_{\text {recovery }}$ and $\mathrm{HR}_{\text {recovery, }}$ respectively.

Note that each of the above-mentioned speech recognition tests had one list of 20 sentences, and none of the sentences was repeated. In addition, the order of the three tested lists that were used in the experiment was randomized across participants. The SPIN score for each tested condition was computed as the ratio between the number of the correctly recognized words and the total number of words contained in each list of 20 MHINT sentences.

\subsection{Data and statistical analysis}

Results are presented as mean \pm standard error of mean (SEM). ANOVA analysis and post-hoc multiplecomparison were performed using SPSS to assess the difference of measured HR and SPIN scores from the measurement sessions with different cardiac activities.

\section{Results}

\subsection{Heart rate at different sessions}

Figure 2 shows the mean HR measured at all sessions during the experiment. First, the mean $\mathrm{HR}_{\text {rest }}$ and $\mathrm{HR}_{\text {training }}$ values are 73.4 and 74.9 beats per minute (bmp), respectively, and the difference between these two values was not significant $(p>0.05)$, suggesting that 
(a)

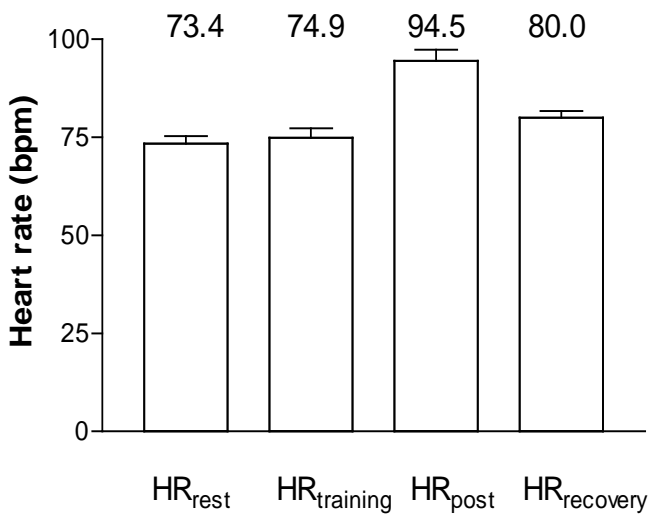

(b)

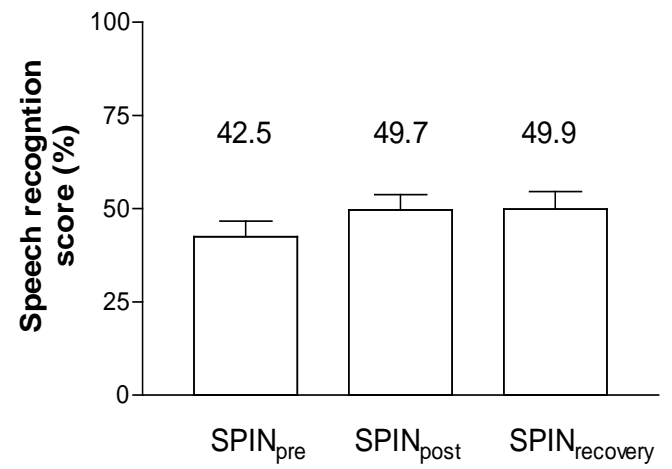

Figure 2. The mean \pm SEM of heart rates (a) and SPIN scores (b) measured across all the sessions.

the immediate exposure to speech-in-noise did not cause significant change in participants' HR.

Secondly, as expected, after a moderate level of exercise (i.e., 5- to 7-min bicycle-riding), the mean HR (i.e., $\mathrm{HR}_{\text {post}}$ ) was significantly increased to $94.5 \mathrm{bmp}$ $(p<0.05)$. Finally, after 10 -min rest, the mean HR (i.e., $\mathrm{HR}_{\text {recovery }}$ ) was reduced towards the value before exercise, i.e., $80.0 \mathrm{bmp}$.

\subsection{SPIN score at different sessions}

Figure 2 (b) shows the effect of autonomic cardiac modulation on SPIN. Before exercise, the mean score of SPIN (i.e., SPIN $\mathrm{Nre}_{\text {) }}$ was $42.5 \%$. However, after a moderate level of exercise, SPIN $_{\text {post }}$ was significantly improved to $49.7 \%(p<0.05)$.

Furthermore, after 10-min rest, although the mean HR was reduced towards the value before exercise, the

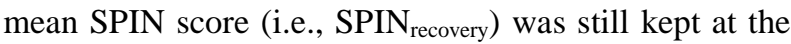
improved level, i.e., $49.9 \%$.

\section{Discussion and conclusion}

The present study quantitatively assessed how the autonomic cardiac activity affected speech perception performance. Specifically, the cardiac activity was modulated by a moderate level of exercise, yielding an increased HR, and by having rest to reduce HR.

Our results showed that the increase of HR was accompanied with the improved performance of speech perception in noise, suggesting that the effect of exercise is two-fold, i.e., increasing both HR and SPIN score. Sympathetic cardiac modulation increases HR and expedites blood flow in human body, which might contribute to improved SPIN performance after moderate exercise.

After a period of recovery, although HR was recovered to a normal resting cardiac activity, the SPIN performance was still better than baseline, suggesting that the effect of autonomic cardiac modulation on SPIN performance may retain for a long period, at least 10 mins as shown in this study. Further work is required regarding how long this improved SPIN performance may sustain. Also, the underlying mechanism for the improved SPIN after recovery needs further investigation.

In summary, the present study quantitatively demonstrated the improved SPIN performance immediately after exercise and recovery, which would help the future investigation on the underlying mechanism of the interaction between autonomic cardiac activity and SPIN.

\section{Acknowledgement}

This work was supported by the National Natural Science Foundation of China (Grant No. 61571213).

\section{References}

[1] Chen F, Li JF, Wong LLN, Yan YH. Effect of linguistic masker on the intelligibility of Mandarin sentences. Proceedings of 15th Annual Conference of the International Speech Communication Association (InterSpeech) 2013; 499-503.

[2] Tolentino J C, Schmidt S L. Association between depression severity and cardiac autonomic modulation. Journal of Psychosomatic Research 2016; 85: 9-11.

[3] Voulgari C, Pagoni S, Vinik A, Poirier P. Exercise improves cardiac autonomic function in obesity and 
diabetes. Metabolism 2013; 62: 609-21.

[4] Glos M, Fietze I, Blau A, Baumann G, Penzel T. Cardiac autonomic modulation and sleepiness: Physiological consequences of sleep deprivation due to $40 \mathrm{~h}$ of prolonged wakefulness. Physiology \& Behavior 2014; 125: 45-53.

[5] Recio A, Linares C, Banegas J R, J Díaz. The short-term association of road traffic noise with cardiovascular, respiratory, and diabetes-related mortality. Environmental Research 2016; 150: 383-90.

[6] Karino S, Usami S, Kumakawa K, et al. Discrimination of Japanese monosyllables in patients with high-frequency hearing loss, Auris Nasus Larynx 2016; 43: 269-80.

[7] Ossmy O, Fried I, Mukamel O. Decoding speech perception from single cell activity in humans. NeuroImage 2015; 117: 151-9.

[8] Stacey P C, Kitterick P T, Morris S D, Sumner C J. The contribution of visual information to the perception of speech in noise with and without information temporal fine structure. Hearing Research 2016; 336: 17-28.
[9] Wong L. L., Soli S. D., Liu S., Han N., Huang M. W. Development of the Mandarin hearing in noise Test (MHINT). Ear and Hearing 2007; 28: 70S-74S.

Address for correspondence:

Fei Chen, Ph. D.

Department of Electrical and Electronic Engineering Southern University of Science and Technology

Xueyuan Road 1088\#, Xili, Nanshan District, Shenzhen, China

Email: fchen@sustc.edu.cn 